\title{
Clinical Characteristics and Outcomes of
}

\section{Hypertensive Patients Infected with COVID-19: A Retrospective Study}

\author{
Liqin Chen ${ }^{1, *}$ \\ Jiankun Chen ${ }^{1,2, *}$ \\ Yuwan $\mathrm{Wu}^{\prime}$ \\ Jinyao Zhong' \\ Fuzhen Zhou' \\ Yuntao Liu ${ }^{1,2}$ \\ Aiting $\mathrm{Xu}^{3}$ \\ Jiqiang $\mathrm{Li}^{1,2}$ \\ Huayang Cai'
}

'The Second Affiliated Hospital (Guangdong Provincial Hospital of Chinese Medicine), Guangzhou University of Chinese Medicine, Guangzhou, 5I 0006, Guangdong, People's Republic of China; ${ }^{2}$ Guangzhou Key Laboratory of Traditional Chinese Medicine for Prevention and Treatment of Emerging Infectious Diseases, Guangzhou, 510006, Guangdong, People's Republic of China; ${ }^{3}$ The People's Hospital of Yangjiang, Yangjiang, 529500,

Guangdong, People's Republic of China

*These authors contributed equally to this work
Background: Hypertension has been reported as the most prevalent comorbidity in patients with coronavirus disease 2019 (COVID-19). This retrospective study aims to compare the clinical characteristics and outcomes in COVID-19 patients with or without hypertension.

Methods: A total of 944 hospitalized patients with laboratory-confirmed COVID-19 were included from January to March 2020. Information from the medical record, including clinical features, radiographic and laboratory results, complications, treatments, and clinical outcomes, were extracted for the analysis.

Results: A total of 311 (32.94\%) patients had comorbidity with hypertension. In COVID-19 patients with hypertension, the coexistence of type 2 diabetes ( $56.06 \%$ vs $43.94 \%$ ), coronary heart disease $(65.71 \%$ vs $34.29 \%)$, poststroke syndrome $(68.75 \%$ vs $31.25 \%)$ and chronic kidney diseases $(77.78 \%$ vs $22.22 \%)$ was significantly higher, while the coexistence of hepatitis B infection (13.04\% vs 86.96\%) was significantly lower than in COVID-19 patients without hypertension. Computed tomography (CT) chest scans show that COVID-19 patients with hypertension have higher rates of pleural effusion than those without hypertension $(56.60 \%$ vs $43.40 \%)$. In addition, the levels of blood glucose [5.80 (IQR, 5.05-7.50) vs 5.39 (IQR, 4.81-6.60)], erythrocyte sedimentation rate (ESR) [28 (IQR, 17.1-55.6) vs 21.8 (IQR, 11.5-44.1), $\mathrm{P}=0.008$ ], C-reactive protein (CRP) [17.92 (IQR, 3.11-46.6) vs 3.15 (IQR, 3.11-23.4), $\mathrm{P}=0.013$ ] and serum amyloid A (SAA) [99.28 (IQR, 8.85-300) vs 15.97 (IQR, 5.97-236.1), $\mathrm{P}=0.005]$ in COVID-19 patients with hypertension were significantly higher than in patients without hypertension.

Conclusion: It is common for patients with COVID-19 to have the coexistence of hypertension, type 2 diabetes, coronary heart disease and so on, which may exacerbate the severity of COVID-19. Therefore, optimal management of hypertension and other comorbidities is essential for better clinical outcomes.

Keywords: COVID-19, coronavirus disease, hypertension, clinical characteristics, comorbidities

\section{Introduction}

The coronavirus disease (COVID-19), caused by the severe acute respiratory syndrome coronavirus 2 (SARS-CoV-2), has become a public health emergency worldwide. It typically presents with fever, cough, myalgia, and fatigue. As of July 22, 2021, there have been 191,773,590 confirmed cases and 4127,963 deaths, with the numbers still rising across 217 countries/regions worldwide. ${ }^{1}$ Therefore, COVID-19 has been considered a pandemic. ${ }^{2}$
Correspondence: Jiqiang Li; Huayang Cai Email lijiqiangjizhen@।63.com;

caihuayang@gzucm.edu.cn 
Previous studies have suggested that the presence of comorbidities among COVID-19 may be associated with poor clinical outcomes. ${ }^{3}$ According to the latest reports, around $20-51 \%$ of patients with COVID-19 have at least one comorbidity, ${ }^{4-6}$ with hypertension $(16.9 \%)$ and diabetes mellitus $(8.2 \%)$ being the most prevalent comorbidities. $^{7}$ A pooled analysis has indicated that hypertension may be related to a higher risk of severe or fatal COVID-19, especially in the elderly. ${ }^{8}$

The angiotensin-converting enzyme 2 (ACE2) has been discovered to be the receptor that allows the entry of SARS-CoV-2 into human cells. ${ }^{9,10}$ ACE2 converts angiotensin II to Ang-(1-7), transforming the vasoconstrictor into a vasodilator peptide, which promotes the release of nitric oxide, decreases the outflow of sympathetic nerves, increases baroreflex sensitivity, and ultimately reduces hypertension. ${ }^{11}$ However, the relationship between COVID-19 and hypertension remains unclear.

The objective of this study was to elucidate the risk and severity of COVID-19 among patients with comorbid hypertension by investigating the clinical features, radiographic and laboratory results, complications, treatments, and clinical outcomes of COVID-19 patients with or without hypertension.

\section{Materials and Methods}

\section{Data Sources}

This was a retrospective study. We included 944 hospitalized COVID-19 patients from the Hubei Provincial Hospital of Integrated Traditional Chinese and Western Medicine from January 2020 to March 2020. All patients confirmed to have COVID-19 using a real-time reversetranscriptase polymerase chain reaction (RT-PCR) assay of the sputum, throat swab, or lower respiratory tract secretion specimens were hospitalized for treatment. All patients were divided into hypertension group and nonhypertension group according to whether they had hypertension or not. Written informed consent was waived owing to the rapid emergence of this infectious disease and the urgent need to collect data.

The demographics, clinical symptoms or signs, radiological and laboratory results, comorbidities, and treatment results were extracted from the electronic medical record system. All medical data reviews and collections were processed by experienced clinicians. We established a computerized database for data storage and verification. Missing or incomplete information was acquired by sending requests for clarification to the coordinators, who subsequently contacted the attending clinicians. Major disagreements during the extraction process were resolved by consultation with the reviewers.

Finally, in accordance with the guidelines for prevention and treatment of hypertension in China (2018 Edition), ${ }^{12}$ we divided the patients into two groups: the COVID-19 with hypertension group and the non-hypertensive group (Hypertension was defined as: clinic systolic $\mathrm{BP} \geq 140$ $\mathrm{mmHg}$ and/or diastolic $\mathrm{BP} \geq 90 \mathrm{mmHg}$ without the use of antihypertensive medications at three visits on different days; with a $\mathrm{BP}<140 / 90 \mathrm{mmHg}$ but having hypertensive history and currently are taking anti-hypertensive medication).

\section{Study Outcomes}

To assess the severity of COVID-19 among the patients with hypertension, the primary clinical outcomes included admission to an intensive care unit (ICU), discharge from the hospital, duration of hospitalization, and death. ${ }^{13}$

\section{Laboratory Confirmation}

Laboratory confirmation of SARS-CoV-2 was performed in accordance with the protocol established by the World Health Organization using RT-PCR assays at Hubei Provincial Hospital of Integrated Traditional Chinese and Western Medicine. The laboratory confirmation processes were similar to the ones used in our previous study. ${ }^{14}$

\section{Statistical Analysis}

Continuous variables are described as mean and standard deviation, and categorical variables are expressed as frequencies and percentages. Continuous variables were analyzed using an independent sample $t$-test when normally distributed; otherwise, the Mann-Whitney test was used. Categorical variables were compared using the chi-square test or Fisher's exact test. All statistical analyses were performed using Statistical Package for the Social Sciences version 13.0 software (IBM; Chicago, Illinois, United States). Statistical significance was set at $\mathrm{P}<0.05$.

\section{Results}

\section{Demographic and Clinical Characteristics}

This study included 944 patients with confirmed COVID19 from the Hubei Provincial Hospital of Integrated Chinese and Western Medicine from January 2020 to March 2020. As shown in Tables 1, 311 (32.94\%) patients had hypertension and $633(67.06 \%)$ were nonhypertensive. The median age of the patients was 60 
Table I Clinical Characteristics of the Study Patients with COVID-19

\begin{tabular}{|c|c|c|c|c|}
\hline \multirow[t]{3}{*}{ Characteristic } & \multicolumn{3}{|l|}{ No. (\%) } & \multirow[t]{3}{*}{$P$ value } \\
\hline & Total & Hypertension & Non-Hypertension & \\
\hline & $944(100)$ & 311 (32.94) & $633(67.06)$ & \\
\hline Female sex - no. /total no. (\%) & $338(35.81)$ & $161(32.26)$ & $338(67.74)$ & 0.6377 \\
\hline Age, Median (IQR) - year & $60(50-69)$ & $67(58-74)$ & $56(46-65)$ & $<0.0001$ \\
\hline Fever on admission (IQR) $-{ }^{\circ} \mathrm{C}$ & $36.7(36.5-37.0)$ & $36.7(36.4-37.0)$ & $36.7(36.5-37.0)$ & 0.8626 \\
\hline Highest temperature during hospitalization (IQR) $-{ }^{\circ} \mathrm{C}$ & $37.2(36.9-37.9)$ & $37.2(36.9-38.0)$ & $37.2(36.9-37.9)$ & 0.2767 \\
\hline Systolic pressure on admission - $\mathrm{mmHg}$ & $125(110-135)$ & $130(120-144)$ & $120(110-131)$ & $<0.000$ I \\
\hline Diastolic pressure on admission $-\mathrm{mmHg}$ & $80(71-90)$ & $82(75-90)$ & $80(70-90)$ & $<0.0001$ \\
\hline \multicolumn{5}{|l|}{ Symptoms - no, \% } \\
\hline Chill & $75(7.94)$ & $26(34.67)$ & $49(63.33)$ & 0.7409 \\
\hline Cough & $535(56.67)$ & $17 \mid(31.96)$ & $364(68.04)$ & 0.4627 \\
\hline Sputum production & $202(21.40)$ & $66(32.67)$ & $136(67.33)$ & 0.9262 \\
\hline Hemoptysis & $6(0.64)$ & $3(50)$ & $3(50)$ & 0.4018 \\
\hline Sore throat & $29(3.07)$ & $9(31.03)$ & $20(68.97)$ & $0.824 I$ \\
\hline Dry throat & I8 (2.54) & $7(38.89)$ & II (6I.II) & 0.3985 \\
\hline Itchy throat & $6(0.82)$ & I (I6.67) & $5(83.33)$ & 0.4774 \\
\hline Nasal congestion & $9(0.95)$ & $2(22.22)$ & 7 (77.78) & 0.4916 \\
\hline Headache & $35(3.7 I)$ & $12(34.29)$ & $23(65.7 I)$ & 0.8634 \\
\hline Shortness of breath & $272(28.8 I)$ & $99(36.40)$ & $173(63.60)$ & 0.1511 \\
\hline Dyspnea & $32(4.35)$ & $9(28.13)$ & $23(71.88)$ & 0.8234 \\
\hline Conjunctival congestion & $4(0.42)$ & I (25.00) & $3(75.00)$ & 0.7248 \\
\hline Chest tightness & $172(23.37)$ & $54(31.40)$ & $118(68.60)$ & 0.6226 \\
\hline Chest pain & $22(2.99)$ & $7(31.82)$ & $15(68.18)$ & 0.8411 \\
\hline Palpitation & $32(4.35)$ & $4(12.50)$ & $28(87.50)$ & 0.0280 \\
\hline Nausea or vomiting & $4 \mathrm{I}(4.34)$ & $12(29.27)$ & $29(70.73)$ & 0.6086 \\
\hline Diarrhea & $69(7.31)$ & $27(39.13)$ & $42(60.87)$ & 0.2562 \\
\hline Myalgia or arthralgia & $125(13.24)$ & $47(37.60)$ & $78(62.40)$ & 0.2345 \\
\hline Mental fatigue & $414(56.25)$ & $124(29.95)$ & $290(70.05)$ & 0.9676 \\
\hline Loss of appetite & $451(61.28)$ & $|4|(3 \mid .26)$ & $310(68.74)$ & 0.3062 \\
\hline Sleep disorder & $435(59.10)$ & $135(31.03)$ & $300(68.97)$ & 0.4154 \\
\hline Fatigue & $604(63.98)$ & $198(32.78)$ & $406(67.22)$ & 0.8868 \\
\hline \multicolumn{5}{|l|}{ Coexisting disorder - no. (\%) } \\
\hline Type 2 diabetes & $132(13.98)$ & $74(56.06)$ & $58(43.94)$ & $<0.0001$ \\
\hline Coronary heart disease & $70(7.42)$ & $46(65.7 I)$ & $24(34.29)$ & $<0.0001$ \\
\hline Poststroke syndrome & $32(3.39)$ & $22(68.75)$ & $10(31.25)$ & $<0.0001$ \\
\hline Malignant tumor & $26(2.75)$ & $5(19.23)$ & $21(80.77)$ & 0.1304 \\
\hline Chronic kidney disease & $9(0.95)$ & 7 (77.78) & $2(22.22)$ & 0.007 \\
\hline Chronic obstructive pulmonary disease & $13(1.38)$ & $4(30.77)$ & $9(69.23)$ & 0.8665 \\
\hline Asthma & $6(0.82)$ & $3(50)$ & $3(50)$ & 0.28 \\
\hline Heart faliure & $6(0.82)$ & $3(50)$ & $3(50)$ & 0.28 \\
\hline Arrhythmia & $18(2.54)$ & $9(50)$ & $9(50)$ & 0.06 \\
\hline Bronchiectasis & $2(0.27)$ & I (50) & $\mathrm{I}(50)$ & 0.5088 \\
\hline Hepatitis B infection* & $23(2.44)$ & $3(13.04)$ & $20(86.96)$ & 0.0431 \\
\hline
\end{tabular}

Notes: *The presence of hepatitis B infection was defined as a positive result upon testing for the hepatitis B surface antigen, with or without elevated levels of alanine or aspartate aminotransferase. Arrhythmias refers to atrial fibrillation, frequent ventricular premature beats, and nodal tachycardia. 
years (IQR, 50-69 years). A total of $35.81 \%$ of the participants were female. Fever (body temperature $\geq 37.5{ }^{\circ} \mathrm{C}$ ) was present in $7.85 \%$ of the patients on admission, increasing to $33.16 \%$ during hospitalization. The most common symptoms were fatigue $(63.98 \%)$, loss of appetite (61.28\%), poor sleep quality (59.10\%), cough (56.67\%), and mental fatigue (56.25\%). Among the overall population, $29.13 \%$ had at least one coexisting illness, including diabetes, poststroke syndrome, malignant tumor, and chronic kidney disease.

The mean age of the hypertensive patients was higher than that of the non-hypertensive patients (67 years [IQR, 58-74] vs 56 years [IQR, 46-65], $\mathrm{P}<0.001$ ), consistent with previous reports. ${ }^{15}$ Furthermore, the systolic $(130 \mathrm{mmHg}$ [IQR, 120144] vs $120 \mathrm{mmHg}$ [IQR, 110-131], $\mathrm{P}<0.001)$ and diastolic blood pressures (82 mmHg [IQR, 75-90] vs $80 \mathrm{mmHg}$ [IQR, 70-90], $\mathrm{P}<0.001$ ) were significantly higher among patients with hypertension than among those without. Palpitation $(12.50 \%$ vs $87.50 \%, \mathrm{P}=0.028)$ was the only symptom that was statistically different between the two groups. It is possible that hypertensive patients were more likely to regularly take antihypertensive medication, making them less sensitive to palpitations.

Table 1 also shows significant differences in the comorbidities between COVID-19 patients with and without hypertension, including diabetes mellitus $(56.06 \%$ vs $43.94 \%$, $\mathrm{P}<0.001)$, coronary heart disease $(65.71 \%$ vs $34.29 \%$, $\mathrm{P}<0.001)$, poststroke syndrome $(68.75 \%$ vs $31.25 \%$, $\mathrm{P}<0.001)$, chronic kidney disease $(77.78 \%$ vs $22.22 \%$, $\mathrm{P}=0.007)$, and hepatitis $\mathrm{B}$ infection $(13.04 \%$ vs $86.96 \%$, $\mathrm{P}=0.043$ ). However, malignant tumors, chronic obstructive pulmonary disease, asthma, heart failure, arrhythmia, and bronchiectasis were similar between the two groups.

\section{Radiologic and Laboratory Findings}

The radiologic indices (Table 2) showed that 767 (81.25\%) of the patients had radiological abnormalities, including
572 cases $(64.41 \%)$ with ground-glass opacity, $76(8.56 \%)$ with local patchy shadowing, $521(58.67 \%)$ with bilateral patchy shadowing, and 53 (5.97\%) with pleural effusion, which are the typical features of an organizing pneumonia pattern of a lung injury. ${ }^{16}$ Compared with nonhypertensive patients, hypertensive patients had higher rates of pleural effusion $(56.60 \%$ vs $43.40 \%, \mathrm{P}<0.001)$, which may have resulted from bacterial superinfection or another cause among the hypertensive COVID-19 patients.

Table 3 shows the laboratory findings on admission. Blood glucose levels $(5.80 \mathrm{mmol} / \mathrm{L}$ [IQR, 5.05-7.50] vs $5.39 \mathrm{mmol} / \mathrm{L}$ [IQR, 4.8-6.60]) in COVID-19 patients with hypertension were significantly higher than those in patients without hypertension $(\mathrm{P}=0.007)$. The hypertensive patients did not have disorders in glucose metabolism because the HbA1c levels were within the normal range. On admission, the white cell count $(5.97 \mathrm{~g} / \mathrm{L}$ [IQR, 4.74-7.67] vs $5.48 \mathrm{~g} / \mathrm{L}$ [IQR, 4.30-6.82]) in COVID-19 patients with hypertension were significantly higher than that in those without hypertension $(\mathrm{P}<0.001)$, while the hemoglobin count was higher in COVID-19 patients without hypertension $(125.6 \mathrm{~g} / \mathrm{L}$ [IQR, 114.5-136] vs $127 \mathrm{~g} / \mathrm{L}$ [IQR, 116-138]; P=0.0316). However, since the parameters were within the normal range, no clinically significant effects were noted.

The levels of the inflammatory markers, including the erythrocyte sedimentation rate $(28 \mathrm{~mm} / \mathrm{h}$ [IQR, 17.1-55.6] vs $21.8 \mathrm{~mm} / \mathrm{h}$ [IQR, 11.5-44.1], $\mathrm{P}=0.008$ ) and C-reactive protein level (17.92 mg/L [IQR, 3.11-46.6] vs $3.15 \mathrm{mg} / \mathrm{L}$ [IQR, 3.11-23.4], $\mathrm{P}=0.013$ ), were elevated, but there were no significant differences between the groups. This suggests that COVID-19 patients with hypertension were more likely to develop more severe infections. Meanwhile, the proinflammatory cytokines, such as procalcitonin, tumor necrosis factor, and interleukins, did not change in either COVID-19 group. The levels of immunity parameters showed that the serum concentrations of $\operatorname{IgE}(50.15 \mathrm{~g} / \mathrm{L}$ [IQR, 18.5-142.5] vs $38.7 \mathrm{~g} / \mathrm{L}$ [IQR, 18.3-95.8]) were

Table 2 Radiographic Examination Results

\begin{tabular}{|l|l|l|l|l|}
\hline Variable- No./Total No. (\%) & Total & Hypertension & Non-Hypertension & P value \\
\cline { 2 - 5 } & $\mathbf{9 4 4 ( \mathbf { 1 0 0 } )}$ & $\mathbf{3 I I ~ ( 3 2 . 9 4 )}$ & $\mathbf{6 3 3}(\mathbf{6 7 . 0 6})$ \\
\hline Abnormalities on chest CT & & & & \\
Ground-glass opacity & $572(64.4 \mathrm{I})$ & $195(34.09)$ & $377(65.9 I)$ & 0.3026 \\
Local patchy shadowing & $76(8.56)$ & $25(32.89)$ & $5 I(67.1 \mathrm{I})$ & 0.9982 \\
Bilateral patchy shadowing & $521(58.67)$ & $183(35.12)$ & $338(64.88)$ & 0.0902 \\
Pleural effusion & $53(5.97)$ & $30(56.60)$ & $23(43.40)$ & 0.0002 \\
\hline
\end{tabular}


Table 3 Laboratory Findings

\begin{tabular}{|c|c|c|c|c|}
\hline \multirow[t]{3}{*}{ Variable- No./Total No. (\%) } & \multicolumn{3}{|l|}{ No. (\%) } & \multirow[t]{3}{*}{$P$ value } \\
\hline & Total & Hypertension & Non-Hypertension & \\
\hline & $944(100)$ & $311(32.94)$ & $633(67.06)$ & \\
\hline \multicolumn{5}{|l|}{ Blood glucose and lipids } \\
\hline Random blood glucose - $\mathrm{mmol} / \mathrm{L}$ & $5.53(4.88-6.94)$ & $5.80(5.05-7.50)$ & $5.39(4.81-6.60)$ & 0.0066 \\
\hline HbAlc - \% & $2(2-5.2)$ & $2(2-3)$ & $2(2-5.3)$ & 0.4810 \\
\hline $\mathrm{TC}-\mathrm{mmol} / \mathrm{L}$ & $4.22(3.64-4.81)$ & $4.19(3.64-4.73)$ & $4.23(3.66-4.67)$ & 0.7850 \\
\hline TG - mmol/L & $1.30(0.94-1.72)$ & $1.33(0.95-1.81)$ & $1.27(0.93-1.70)$ & 0.3223 \\
\hline LDL-C - mmol/L & $1.79(1.46-2.17)$ & $1.77(1.50-2.18)$ & $1.80(1.42-2.17)$ & 0.8271 \\
\hline $\mathrm{HDL}-\mathrm{C}-\mathrm{mmol} / \mathrm{L}$ & $1.10(0.93-1.30)$ & $1.12(0.89-0.23)$ & $1.10(0.95-1.30)$ & 0.3518 \\
\hline \multicolumn{5}{|l|}{ Hematologic } \\
\hline White cell count - g/L & $5.61(4.47-7.09)$ & $5.97(4.74-7.67)$ & $5.48(4.3-6.82)$ & 0.0008 \\
\hline Lymphocyte count - g/L & $1.32(0.88-1.77)$ & $1.28(0.83-1.72)$ & $1.36(0.91-1.82)$ & 0.2339 \\
\hline Platelet count - g/L & $220(169-277)$ & $221(164-288)$ & $218.5(|7|-272)$ & 0.2809 \\
\hline Hemoglobin - $g / L$ & $127(116-137)$ & $125.6(114.5-136)$ & $127(116-138)$ & 0.0316 \\
\hline \multicolumn{5}{|l|}{ Inflammatory } \\
\hline ESR - mm/h & $25(13.5-48.1)$ & $28(17.1-55.6)$ & $21.8(11.5-44.1)$ & 0.0083 \\
\hline TNF - fmol/mL & $9.3(6.8-12.7)$ & $9.9(7.03-14.2)$ & $9.3(6.49-12.1)$ & 0.7094 \\
\hline IL-I $\beta-p g / m L$ & $5(5-5.8)$ & $5(5-5.8)$ & $5(5-5.72)$ & 0.3926 \\
\hline IL-I0 - pg/mL & $5(5-7.2)$ & $5(5-7.8)$ & $5(5-6.6)$ & 0.1855 \\
\hline $\mathrm{IL}-6$ - pg/mL & $5.3(2.6-16.1)$ & $6.02(3.02-18.2)$ & $4.95(2.49-14.8)$ & 0.2482 \\
\hline PCT - ng/mL & $0.03(0.02-0.07)$ & $0.04(0.02-0.08)$ & $0.03(0.02-0.06)$ & 0.5126 \\
\hline $\mathrm{CRP}-\mathrm{mg} / \mathrm{L}$ & $5.85(3.11-37.1)$ & $17.92(3.11-46.6)$ & $3.15(3.11-23.4)$ & 0.0131 \\
\hline \multicolumn{5}{|l|}{ Immune } \\
\hline $\lg A-g / L$ & $2.19(1.84-2.7)$ & $2.22(1.85-2.79)$ & $2.18(1.84-2.61)$ & 0.1964 \\
\hline $\lg M-g / L$ & $0.99(0.79-1.28)$ & $0.89(0.68-1.15)$ & $1.03(0.85-1.34)$ & $<0.0001$ \\
\hline $\lg G-g / L$ & $11.7(10.3-13.3)$ & $11.9(10.8-13.2)$ & $11.7(10.2-13.4)$ & 0.4699 \\
\hline $\lg E-g / L$ & $40.6(18.3-115.0)$ & $50.15(18.50-142.5)$ & $38.7(18.3-95.8)$ & 0.0363 \\
\hline $\mathrm{C} 3-\mathrm{g} / \mathrm{L}$ & $1.18(1.03-1.33)$ & $1.22(1.11-1.37)$ & $1.16(0.98-1.29)$ & 0.5755 \\
\hline $\mathrm{C} 4-\mathrm{g} / \mathrm{L}$ & $0.26(0.21-0.33)$ & $0.27(0.24-0.34)$ & $0.25(0.20-0.32)$ & 0.0196 \\
\hline Total T lymphocytes - \% & 68.1 (59.79-74.2) & $66.43(57.09-73.05)$ & $69.14(61.04-74.65)$ & 0.0633 \\
\hline Total T lymphocytes count & $618(372-821)$ & $546(282-787)$ & $662.5(421-838)$ & 0.0364 \\
\hline Cytotoxic T lymphocytes - \% & $23.30(17.2-29.8)$ & $20.66(15.86-28.16)$ & $24.32(18.36-30.33)$ & 0.0580 \\
\hline Cytotoxic T lymphocytes count & 205 (II8-299) & $170(100-266)$ & $211.5(134-313)$ & 0.1275 \\
\hline Helper T cells - \% & $40.76(33.52-48.18)$ & $41.54(32.94-48.82)$ & $40.04(34.03-47.91)$ & 0.6426 \\
\hline Helper T cells count & $364(225-512)$ & $345.5(167-508)$ & $372(24 I-5 \mid 4)$ & 0.2946 \\
\hline B lymphocytes - \% & $13.68(9.88-19.22)$ & $13.50(9.40-19.32)$ & $13.7(10.08-19.04)$ & 0.8565 \\
\hline B lymphocytes count & $112(77-172.5)$ & $103(64.5-151.5)$ & $116(83.5-183.5)$ & 0.0445 \\
\hline K cells - \% & $13.85(8.88-20.34)$ & $15.66(9.94-22.75)$ & $13.26(8.50-19.44)$ & 0.0120 \\
\hline$K$ cells count & $112(65.5-181.5)$ & $116(65.5-187.5)$ & $110.5(65.5-178)$ & 0.5853 \\
\hline Lymphocytes count & $908(609-1167)$ & $851(530-1122)$ & 951 (678-1199) & 0.1020 \\
\hline Helper/Cytotoxic T lymphocytes ratio & $1.78(1.26-2.54)$ & $2.10(1.38-2.66)$ & $1.64(1.19-2.32)$ & 0.2465 \\
\hline \multicolumn{5}{|l|}{ Coagulation } \\
\hline Fibrinogen - $\mathrm{g} / \mathrm{L}$ & $2.91(2.32-3.88)$ & $3.03(2.44-3.97)$ & $2.84(2.25-3.83)$ & 0.0116 \\
\hline PT - s & $12.1(11.3-13.1)$ & $12.1(11.2-13.1)$ & $12.1(11.3-13.1)$ & 0.4645 \\
\hline INR & $1.01(0.94-1.10)$ & $1.01(0.94-1.09)$ & $1.01(0.95-1.10)$ & 0.3082 \\
\hline PAP & $99(94-104)$ & $99(94-104)$ & $99(94-103.8)$ & 0.1569 \\
\hline APTT - s & $30.00(27.7-32.2)$ & $29.45(27.3-32.2)$ & $30.1(27.9-32.2)$ & 0.5937 \\
\hline $\mathrm{TT}-\mathrm{s}$ & 15.75 (|4.85-17.0I) & $15.8(14.9-17.1)$ & $15.7(14.8-17.0)$ & 0.3625 \\
\hline D-dimer - mg/L & $0.48(0.32-0.97)$ & $0.59(0.38-1.30)$ & $0.43(0.29-0.89)$ & 0.3873 \\
\hline
\end{tabular}

(Continued) 
Table 3 (Continued).

\begin{tabular}{|c|c|c|c|c|}
\hline \multirow[t]{3}{*}{ Variable- No./Total No. (\%) } & \multicolumn{3}{|l|}{ No. (\%) } & \multirow[t]{3}{*}{$P$ value } \\
\hline & Total & Hypertension & Non-Hypertension & \\
\hline & $944(100)$ & 311 (32.94) & $633(67.06)$ & \\
\hline \multicolumn{5}{|l|}{ Biochemical } \\
\hline BNP - pg/mL & $26(10-64)$ & $37.5(|3-9|)$ & $23(10-50.5)$ & 0.0042 \\
\hline ALT - IU/L & $20(11-35)$ & $20(12-35)$ & $20(11-35.5)$ & 0.4357 \\
\hline AST - IU/L & $22(16-32)$ & $23(16-35)$ & $21(|6-3|)$ & 0.3370 \\
\hline TP- $g / L$ & $63.6(59.5-67.5)$ & $63.75(60.1-67.9)$ & $63.45(59.3-67.1)$ & 0.3625 \\
\hline ALB- $g / L$ & $37.85(34.3-41.2)$ & $37.4(34.0-40.3)$ & $38.1(34.5-4 \mid .4)$ & 0.1262 \\
\hline PA- $g / L$ & $153.7(|06.2-2| 1.5)$ & $154.7(103.1-219.2)$ & $153.6(107.7-206.5)$ & 0.5847 \\
\hline GLB- $g / L$ & $25.8(22.4-29.01)$ & $26.1(23.3-29.5)$ & $25.5(22.0 \mathrm{I}-28.8)$ & 0.6356 \\
\hline Cys-C - mg/L & $0.79(0.66-0.98)$ & $0.90(0.74-1.16)$ & $0.75(0.64-0.89)$ & $<0.0001$ \\
\hline TBIL $-\mu \mathrm{mol} / \mathrm{L}$ & II (8.2-14.6) & II.5 (8.4-15.8) & $10.9(8.2-14.1)$ & 0.0578 \\
\hline CRE - $\mu \mathrm{mol} / \mathrm{L}$ & $66.3(56.8-81.3)$ & $72.75(60.4-86.4)$ & $64(55.2-78.2)$ & $<0.0001$ \\
\hline BUN- mmol/L & $4.76(3.76-6.01)$ & $5.16(4.08-6.65)$ & $4.53(3.67-5.72)$ & 0.0741 \\
\hline UA- $\mathrm{mmol} / \mathrm{L}$ & $269.7(219.7-340.1)$ & $290.3(237.4-365.8)$ & $260.5(213.5-321.7)$ & $<0.0001$ \\
\hline Creatinine $-\mu \mathrm{mol} / \mathrm{L}$ & $63(39-104.5)$ & $64(42-105)$ & $62(38-103)$ & 0.9357 \\
\hline $\mathrm{LDH}-\mathrm{U} / \mathrm{L}$ & $210(170.5-278.0)$ & $219(180-298)$ & $204(168-266)$ & 0.3137 \\
\hline SAA- mg/L & $22.18(6.02-254.04)$ & $99.28(8.85-300)$ & $15.97(5.97-236.1)$ & 0.0050 \\
\hline \multicolumn{5}{|l|}{ Blood Gas Analysis } \\
\hline $\mathrm{PH}$ & $7.45(7.42-7.48)$ & $7.45(7.42-7.48)$ & $7.45(7.42-7.48)$ & 0.5964 \\
\hline $\mathrm{P}_{\mathrm{CO} 2}-\mathrm{mmHg}$ & $36(33-4 I)$ & $35(33-4 I)$ & $37(32.5-4 I)$ & 0.6320 \\
\hline $\mathrm{P}_{\mathrm{O} 2}-\mathrm{mmHg}$ & $94(69-123)$ & $85(70-113)$ & $98.5(68.5-134)$ & 0.0264 \\
\hline \multicolumn{5}{|l|}{ Minerals } \\
\hline Sodium - mmol/L & $139.7(|37.4-14| .3)$ & $139.5(|37-| 4 \mid .3)$ & $139.8(137.5-141.3)$ & 0.2377 \\
\hline Potassium - mmol/L & $3.82(3.5 \mid-4.2 I)$ & $3.77(3.42-4.21)$ & $3.85(3.55-4.20)$ & 0.1796 \\
\hline Chloride - $\mathrm{mmol} / \mathrm{L}$ & $106(103.6-107.7)$ & $105.7(102.8-107.4)$ & $106.0(103.7-107.8)$ & 0.0488 \\
\hline
\end{tabular}

Abbreviations: HbAlc, glycosylated hemoglobin AI; TC, total cholesterol; TG, triglyceride; LDL-C, low-density lipoprotein cholesterol; HDL-C, high-density lipoprotein cholesterol; ESR, erythrocyte sedimentation rate; CRP, C-reactive protein; PCT, procalcitonin; TNF, tumor necrosis factor; IL, interleukin; BUN, blood urea nitrogen; PT, prothrombin time; INR, international normalized ratio; APTT, activated partial thromboplastin time; PAP, prothrombin activation percentage; TT, thrombin time; LDH, lactic dehydrogenase; Ig, immunoglobulin; C3, complement 3; C4, complement 4; AST, aspartate aminotransferase; ALT, alanine aminotransferase; SAA, serum amyloid A; PH, pondus hydrogenii.

increased $(\mathrm{P}=0.036)$, whereas the serum levels of complement $4(0.27 \mathrm{~g} / \mathrm{L}[\mathrm{IQR}, 0.24-0.34]$ vs $0.25 \mathrm{~g} / \mathrm{L}$ [IQR, 0.20 0.32]) were decreased $(\mathrm{P}=0.020)$ in the hypertensive COVID-19 patients when compared with the nonhypertensive COVID-19 patients. The level of complement 4 was decreased in both groups, indicating viral infection. The IgM level, total T lymphocyte count, B lymphocyte count, and percentage of $\mathrm{K}$ cells showed positive changes, but no clinical manifestations were observed.

Compared to the COVID-19 group without hypertension, the COVID-19 group with hypertension had a higher level of fibrinogen, though its level was within the normal range in both groups. The levels of D-dimer, activated partial thromboplastin time, thrombin time, etc. were within the laboratory ranges, suggesting a more or less normal blood coagulation function. Serum amyloid A levels were higher in the hypertensive group than in the non-hypertensive group (99.28 mg/L [IQR, 8.85-300] vs $15.97 \mathrm{mg} / \mathrm{L}$ [IQR, 5.97236.1], $\mathrm{P}=0.005$ ), suggesting acute infection. The level of the brain natriuretic peptide showed a positive change, but it was clinically irrelevant. Although cystatin C, creatinine, and uric acid levels of both the hypertensive and non-hypertensive groups were within normal range, the hypertensive patients had higher cystatin $\mathrm{C}(0.90 \mathrm{mg} / \mathrm{L}$ [IQR, 0.74-1.16] vs $0.75 \mathrm{mg} / \mathrm{L}$ [IQR, 0.64-0.89], $\mathrm{P}<0.001)$, creatinine, and uric acid levels than the non-hypertensive patients, possibly indicating renal function injury. No abnormalities were noted in the levels of alanine aminotransferase, aspartate aminotransferase, and blood urea nitrogen. Compared to the nonhypertensive patients, the hypertensive patients had much lower PO2 (85 mmHg [IQR, 70-113] vs $98.5 \mathrm{mmHg}$ [IQR, 68.5-134], $\mathrm{P}=0.026$ ). This may be related to the ACE2 levels, 
as it has been previously reported that hypoxia could decrease ACE2 expression in the lung tissues of rats. ${ }^{17}$

\section{Clinical Outcomes}

As shown in Table 4, 86 patients were admitted to the ICU and 904 patients were discharged from the hospital. There were no significant differences in the incidence of ICU admission and hospital discharge between the two groups. No deaths were observed during the entire study. The median duration of hospitalization was 14 days (median, 9-20 days). Patients with hypertension had significantly longer hospital stays (14 [IQR, 9-21] vs 13 [IQR, 8-19], $\mathrm{P}=0.022)$ than those without hypertension.

\section{Treatment and Complications}

All COVID-19 patients received symptomatic treatment. The majority of the patients $(77.67 \%)$ received antiviral therapy, while $61.5 \%$ received intravenous antibiotic therapy. Furthermore, $31.31 \%$ received systemic glucocorticoids, and $1.44 \%$ received antifungal medication. The majority $(89.62 \%)$ received oxygen therapy, while only a few required invasive (1.52\%) and noninvasive mechanical ventilation (7.16\%). In addition, $83.93 \%$ received traditional Chinese medicine treatment, $69.92 \%$ received Chinese patent medicine treatment, and $16.68 \%$ received acupuncture or ear acupoint therapy. There were no significant differences in the above treatment measures between the COVID-19 patients with and without hypertension. Anti-hypertensive drugs and lipid-lowering drugs were administered more to patients with hypertension than to those without $(62.12 \%$ vs $37.88 \%, \mathrm{P}<0.001$ and $44.00 \%$ vs $56.00 \%, \mathrm{P}<0.036$, respectively), whereas antidiabetic drugs were used less frequently in hypertensive patients (44.00\% vs $56.00 \%, \mathrm{P}=0.036$ ) (Table 4).

Table 4 Complications, Treatments, and Clinical Outcomes

\begin{tabular}{|c|c|c|c|c|}
\hline \multirow[t]{3}{*}{ Variable } & No. (\%) & & & \multirow[t]{3}{*}{$P$ value } \\
\hline & Total & Hypertension & Non-Hypertension & \\
\hline & $944(100)$ & 31 I (32.94) & 633(67.06) & \\
\hline \multicolumn{5}{|l|}{ Complications } \\
\hline Septic shock - no. (\%) & $10(1.07)$ & $2(20.00)$ & $8(80.00)$ & 0.3922 \\
\hline Heart failure - no. (\%) & $14(1.50)$ & $8(57.14)$ & $6(42.86)$ & 0.0799 \\
\hline Acute respiratory distress syndrome - no. (\%) & $48(5.15)$ & $15(31.25)$ & $33(68.75)$ & 0.8356 \\
\hline Acute kidney injury - no. (\%) & $10(1.07)$ & $5(50)$ & $5(50)$ & 0.2385 \\
\hline Disseminated intravascular coagulation - no. (\%) & $2(0.2 I)$ & $\mathrm{I}(50)$ & I (50) & 0.5467 \\
\hline Rhabdomyolysis - no. (\%) & $\mathrm{I}(0.1 \mathrm{I})$ & $0(0.00)$ & I $(100.00)$ & 1.0000 \\
\hline \multicolumn{5}{|l|}{ Treatments } \\
\hline Anti-hypertensive medication - no. (\%) & $264(35.87)$ & $164(62.12)$ & $100(37.88)$ & $<0.0001$ \\
\hline Antidiabetic medication - no. (\%) & 50 (6.79) & $22(44.00)$ & $28(56.00)$ & 0.0362 \\
\hline Lipid-lowering medication - no. (\%) & $65(8.83)$ & $40(61.54)$ & $25(38.46)$ & $<0.0001$ \\
\hline Intravenous antibiotics - no. (\%) & $567(61.50)$ & $192(33.86)$ & $375(66.14)$ & 0.2780 \\
\hline Antifungal medication - no. (\%) & $13(1.44)$ & $6(46.15)$ & $7(53.85)$ & 0.2747 \\
\hline Antiviral medication - no. (\%) & $714(76.77)$ & $243(34.03)$ & 47I (65.97) & 0.0561 \\
\hline Systemic glucocorticoids - no. (\%) & $284(31.3 I)$ & $98(34.51)$ & $186(65.49)$ & 0.2912 \\
\hline Oxygen therapy- no. (\%) & $798(85.62)$ & $267(33.46)$ & $531(66.54)$ & 0.1816 \\
\hline Invasive mechanical ventilation - no. (\%) & $14(1.52)$ & $6(42.86)$ & $8(57.14)$ & 0.3940 \\
\hline Noninvasive mechanical ventilation - no. (\%) & $66(7.16)$ & $28(42.42)$ & $38(57.58)$ & 0.0754 \\
\hline Intravenous immunoglobulin- no. (\%) & $227(24.57)$ & $78(34.36)$ & $149(65.64)$ & 0.4336 \\
\hline Traditional Chinese Medicine - no. (\%) & $781(83.93)$ & $245(31.37)$ & $536(68.63)$ & 0.1052 \\
\hline Chinese Patent Medicine - no. (\%) & $651(69.92)$ & $214(32.87)$ & $437(67.13)$ & 0.7029 \\
\hline Acupuncture or ear acupoint - no. (\%) & $154(16.68)$ & $59(38.31)$ & $95(61.69)$ & 0.0856 \\
\hline \multicolumn{5}{|l|}{ Clinical outcomes at data cutoff } \\
\hline Admission to intensive care unit - no. (\%) & $86(9.12)$ & $32(37.21)$ & $54(62.79)$ & 0.3815 \\
\hline Discharge from hospital & $904(95.76)$ & $294(32.52)$ & $610(67.48)$ & 0.1889 \\
\hline Median length of hospital stay (IQR) - days & $13(9-20)$ & $14(9-2 \mid)$ & $13(8-19)$ & 0.0216 \\
\hline
\end{tabular}


During hospital admission, patients were diagnosed with acute respiratory distress syndrome $(5.15 \%)$, acute heart failure $(1.50 \%)$, septic shock $(1.07 \%)$, acute kidney injury $(1.07 \%)$, disseminated intravascular coagulation (DIC) $(0.21 \%)$, and rhabdomyolysis $(0.11 \%)$. However, all the above-mentioned diagnoses showed similar incidences in the two groups.

\section{Discussion}

Compared to the diseases associated with previous coronavirus epidemics, such as the Severe Acute Respiratory Syndrome and the Middle East Respiratory Syndrome, COVID-19 is more severe and has a faster spread. ${ }^{18}$ SARS-CoV-2 enters cells through the ACE2 receptor, considered the functional receptor for this coronavirus. ${ }^{19}$ Additionally, ACE2 is regarded as a potent modulator of blood pressure, and its deficiency leads to hypertension. ${ }^{20}$ Due to the interaction between SARS-CoV-2 and ACE2, it is believed that hypertension may be related to the pathogenesis of COVID-19 by working directly as a previous clinical predictor of disease severity or by leading to late deterioration in the disease process. ${ }^{8}$

Hypertension, diabetes, chronic obstructive pulmonary disease, cardiovascular disease, and cerebrovascular disease are risk factors for COVID-19. ${ }^{21-25}$ In this retrospective cohort study, we focused on hypertension as it was the most prevalent comorbidity among patients with COVID19. The median age of the COVID-19 patients was 60 years, and the prevalence rate of hypertension was $32.94 \%$. We found no difference in the patients' sex distribution, but the patients with hypertension were older and had higher blood pressures, consistent with a previous study. ${ }^{26}$ This may be due to the higher median age of the patients we included. Normally, the frequency of palpitation is higher in patients with hypertension. However, in our study, the incidence of palpitations was lower in the hypertensive group, which could have resulted from the small number of patients with palpitation or the presence of drug interventions in hypertensive patients. Our study indicated that hypertensive COVID19 patients had more comorbidities, such as diabetes mellitus, coronary heart disease, pleural effusion, and chronic kidney disease.

Chest CT images proved that patients with hypertension had higher rates of pleural effusion than those without hypertension, indicating that the former had more severe lung pathologies. ${ }^{27}$ Furthermore, the levels of the inflammatory markers, such as the erythrocyte sedimentation rate, serum amyloid $\mathrm{A}$, and $\mathrm{C}$-reactive protein, were elevated in the COVID-19 patients, pointing to an increased likelihood of developing cytokine storm in these patients. Furthermore, the levels of these markers were significantly higher in patients with hypertension than in those without hypertension. Integrating these results with the chest CT results above, we can surmise that COVID-19 patients with hypertension were more likely to develop a more severe infection. Hence, patients in the hypertensive group had longer hospital stays than those in the nonhypertensive group. Moreover, there was no significant difference in clinical treatment between the two groups, except for the use of antihypertensive and lipid-lowering drugs.

\section{Study Limitations}

Our study had the following limitations. First, this study was retrospective and non-randomized. Thus, the clinical outcomes were not as accurate as those in prospective studies. Second, the severity of COVID-19 was not graded. As such, the proportion of patients with each grade could not be determined. Third, due to the outbreak isolation management and lack of equipment, the blood pressure was not measured daily. Fourth, systemic glucocorticoids have been widely used to treat COVID-19 patients, which may influence blood pressure. Therefore, multicenter and prospective studies are necessary to further explore the risk factors and severe events in COVID-19 patients with hypertension.

\section{Conclusion}

In conclusion, hypertension worsens the morbidity of COVID-19 patients. This study provided at least two essential lessons for the clinical management of COVID19. First, controlling blood pressure in COVID-19 patients with hypertension is necessary. Second, increased clinical vigilance is warranted for COVID-19 patients with hypertension, given its chronicity and associated morbidity.

\section{Ethics Statement}

The study was performed in accordance with the Declaration of Helsinki and carried out in accordance with the recommendations of Chinese National Guidelines and Ethics Branch of the Biomedical Ethics Committee of Guangzhou University of Chinese Medicine (ZE2020-049-01). 


\section{Acknowledgments}

The authors are grateful to the doctors and nurses of Hubei Provincial Integrated Traditional Chinese and Western Medicine Hospital for their assistance.

\section{Author Contributions}

All authors made substantial contributions to conception and design, acquisition of data, or analysis and interpretation of data; took part in drafting the article or revising it critically for important intellectual content; agreed to submit to the current journal; gave final approval of the version to be published; and agree to be accountable for all aspects of the work.

\section{Funding}

This work was supported by the National Key Research and Development Plan of China (2020YFA0708001, 2020YFC0845300, 2020YFC0841600), the Guangdong Province Science and Technology Planning Project (2019A070717007), the Guangdong Provincial Department of Education Project (2020KZDZX1054), the Fund of Guangzhou University of Chinese Medicine (2021XK06, 2021XK49, 2021YJZX012), the Guangdong Provincial Bureau of Chinese Medicine (20212094 and 20191377), and the Guangdong Provincial Hospital of Chinese Medicine Fund (YN2019ML11 and YN2019QJ13).

\section{Disclosure}

The authors declare no conflicts of interest in relation to this work.

\section{References}

1. WHO. WHO Coronavirus disease (COVID-19) dashboard; 2021. Available from: https://covid19.who.int/. Accessed August 13, 2021.

2. Tian J, Yuan X, Xiao J, et al. Clinical characteristics and risk factors associated with COVID-19 disease severity in patients with cancer in Wuhan, China: a multicentre, retrospective, cohort study. Lancet Oncol. 2020;21(7):893-903.

3. Wang D, Hu B, Hu C, et al. Clinical characteristics of 138 hospitalized patients with 2019 novel Coronavirus-infected pneumonia in Wuhan, China. JAMA. 2020;323(11):1061-1069. doi:10.1001/jama.2020.1585

4. Huang $\mathrm{C}$, Wang Y, Li X, et al. Clinical features of patients infected with 2019 novel coronavirus in Wuhan, China. Lancet. 2020;395 (10223):497-506. doi:10.1016/S0140-6736(20)30183-5

5. Chen N, Zhou M, Dong X, et al. Epidemiological and clinical characteristics of 99 cases of 2019 novel coronavirus pneumonia in Wuhan, China: a descriptive study. Lancet. 2020;395 (10223):507-513. doi:10.1016/S0140-6736(20)30211-7

6. Liu K, Fang YY, Deng Y, et al. Clinical characteristics of novel coronavirus cases in tertiary hospitals in Hubei Province. Chin Med $J \quad$ (Engl). 2020;133(9):1025-1031. doi:10.1097/CM9.000000000 0000744
7. Guan WJ, Liang WH, Zhao Y, et al. Comorbidity and its impact on 1590 patients with COVID-19 in China: a nationwide analysis. Eur Respir J. 2020;55(5):2000547. doi:10.1183/13993003.005472020

8. Lippi G, Wong J, Henry BM. Hypertension in patients with coronavirus disease 2019 (COVID-19): a pooled analysis. Pol Arch Intern Med. 2020;130(4):304-309. doi:10.20452/pamw.15272

9. Zhou P, Yang XL, Wang XG, et al. A pneumonia outbreak associated with a new coronavirus of probable bat origin. Nature. 2020;579 (7798):270-273. doi:10.1038/s41586-020-2012-7

10. Hoffmann M, Kleine-Weber H, Schroeder S, et al. SARS-CoV-2 cell entry depends on ACE2 and TMPRSS2 and is blocked by a clinically proven protease inhibitor. Cell. 2020;181(2):271-280.e8. doi:10.1016/j.cell.2020.02.052

11. Xu J, Sriramula S, Xia $\mathrm{H}$, et al. Clinical relevance and role of neuronal AT1 receptors in ADAM17-mediated ACE2 shedding in neurogenic hypertension. Circ Res. 2017;121(1):43-55. doi:10.1161/ CIRCRESAHA.116.310509

12. Joint Committee for Guideline Revision. 2018 Chinese guidelines for prevention and treatment of hypertension-a report of the revision Committee of Chinese Guidelines for prevention and treatment of hypertension. J Geriatr Cardiol. 2019;16(3):182-241. doi:10.11909/j. issn.1671-5411.2019.03.014.

13. Jin X, Pang B, Zhang J, et al. Core outcome set for clinical trials on Coronavirus disease 2019 (COS-COVID). Engineering (Beijing). 2020;6(10):1147-1152. doi:10.1016/j.eng.2020.03.002

14. Chen Y, Chen J, Gong X, et al. Clinical characteristics and outcomes of type 2 diabetes patients infected with COVID-19: a retrospective study. Engineering (Beijing). 2020;6(10):1170-1177. doi:10.1016/j. eng.2020.05.017

15. Grasselli G, Zangrillo A, Zanella A, et al. Baseline characteristics and outcomes of 1591 patients infected with SARS-CoV-2 admitted to ICUs of the Lombardy Region, Italy. JAMA. 2020;323 (16):1574-1581. doi:10.1001/jama.2020.5394

16. Kanne JP, Little BP, Chung JH, Elicker BM, Ketai LH. Essentials for radiologists on COVID-19: an update-radiology scientific expert panel. Radiology. 2020;296(2):E113-E114. doi:10.1148/ radiol.2020200527

17. Hu HH, Zhang RF, Dong LL, Chen EG, Ying KJ. Overexpression of ACE2 prevents hypoxia-induced pulmonary hypertension in rats by inhibiting proliferation and immigration of PASMCs. Eur Rev Med Pharmacol Sci. 2020;24(7):3968-3980. doi:10.26355/ eurrev_202004_20867

18. Datta PK, Liu F, Fischer T, Rappaport J, Qin X. SARS-CoV-2 pandemic and research gaps: understanding SARS-CoV-2 interaction with the ACE2 receptor and implications for therapy. Theranostics. 2020;10(16):7448-7464. doi:10.7150/thno.48076

19. Walls AC, Park YJ, Tortorici MA, Wall A, McGuire AT, Veesler D. Structure, function, and antigenicity of the SARS-CoV-2 spike glycoprotein. Cell. 2020;181(2):281-292.e6. doi:10.1016/j. cell.2020.02.058

20. Patel VB, Zhong JC, Grant MB, Oudit GY. Role of the ACE2/ angiotensin 1-7 axis of the renin-angiotensin system in heart failure. Circ Res. 2016;118(8):1313-1326. doi:10.1161/CIRCRESAHA.1 16.307708

21. Wang B, Li R, Lu Z, Huang Y. Does comorbidity increase the risk of patients with COVID-19: evidence from meta-analysis. Aging (Albany NY). 2020;12(7):6049-6057. doi:10.18632/aging.103000

22. Li M, Dong Y, Wang H, et al. Cardiovascular disease potentially contributes to the progression and poor prognosis of COVID-19. Nutr Metab Cardiovasc Dis. 2020;30(7):1061-1067. doi:10.1016/j. numecd.2020.04.013

23. Katz JM, Libman RB, Wang JJ, et al. Cerebrovascular complications of COVID-19. Stroke. 2020;51(9):e227-e231. doi:10.1161/ STROKEAHA.120.031265 
24. Amar J, Touront N, Ciron AM, Pendaries C. Interactions between hypertension and inflammatory tone and the effect on blood pressure and outcomes in patients with COVID-19. J Clin Hypertens (Greenwich). 2021;23(2):238-244. doi:10.1111/JCH.14137

25. Pena JE, Rascon-Pacheco RA, Ascencio-Montiel IJ, et al. Hypertension, diabetes and obesity, major risk factors for death in patients with COVID-19 in Mexico. Arch Med Res. 2021;52 (4):443-449. doi:10.1016/J.ARCMED.2020.12.002
26. Huang S, Wang J, Liu F, et al. COVID-19 patients with hypertension have more severe disease: a multicenter retrospective observational study. Hypertens Res. 2020;43(8):824-831. doi:10.1038/s41440-0200485-2

27. Ye Z, Zhang Y, Wang Y, Huang Z, Song B. Chest CT manifestations of new coronavirus disease 2019 (COVID-19): a pictorial review. Eur Radiol. 2020;30(8):4381-4389. doi:10.1007/s00330-020-06801-0

\section{Publish your work in this journal}

The International Journal of General Medicine is an international, peer-reviewed open-access journal that focuses on general and internal medicine, pathogenesis, epidemiology, diagnosis, monitoring and treatment protocols. The journal is characterized by the rapid reporting of reviews, original research and clinical studies across all disease areas. The manuscript management system is completely online and includes a very quick and fair peer-review system, which is all easy to use. Visit http://www.dovepress.com/ testimonials.php to read real quotes from published authors. 